\title{
Preferences and differences in the trail pheromone of the leaf-cutting ant Atta sexdens sexdens (Hymenoptera: Formicidae)
}

\author{
E. David MORGAN ${ }^{1}$, SARAH J. KEEGANS ${ }^{1}$, JozeF TITS $^{2}$, Tom WENSELEERS ${ }^{2}$ and Johan BILLEN ${ }^{2}$ \\ ${ }^{1}$ Chemical Ecology Group, Lennard-Jones Laboratory, Keele University, Staffordshire ST5 5BG, UK; \\ e-mail: e.d.morgan@chem.keele.ac.uk \\ ${ }^{2}$ Zoological Institute, Katholieke Universiteit Leuven, Naamsestraat 59, B-3000 Leuven, Belgium; \\ e-mails: tom.wenseleers@bio.kuleuven.be, johan.billen@bio.kuleuven.be
}

Key words. Formicidae, trail pheromone, caste differences, pheromone concentration, trail width, colony specificity, venom gland, Dufour gland, Atta sexdens sexdens

\begin{abstract}
The amount of the trail pheromone substance, 3-ethyl-2,5-dimethylpyrazine, of the leaf-cutting ant Atta sexdens sexdens (Hymenoptera: Formicidae) increases with increasing size of the ant from 0 to $35 \mathrm{ng}$ per individual. The compounds 2,5-dimethylpyrazine and trimethylpyrazine are also consistently present. Trails of venom gland extracts are followed with unequal fidelity by different castes. Minor workers follow best, mediums least well. Workers walk sinuously on narrow trails and less sinuously on wider trails up to $9 \mathrm{~mm}$. Trails wider than $9 \mathrm{~mm}$ are not followed. The optimum concentration of pheromone on synthetic trails lies between 15 and $150 \mathrm{pg} \mathrm{cm}^{-1}$. Given a choice of concentrations at a trail branch, workers always choose the more concentrated. Workers showed slight preference for a trail made with venom gland secretion from their own, over that from an alien colony, but there is no preference for a trail that contains Dufour gland secretion additionally.
\end{abstract}

\section{INTRODUCTION}

Bonnet (1779) first recorded that some ants use trails to recruit workers to a food source. Gradually our knowledge of these trails and the behaviour of ants applying and following them have advanced. With the advent of sufficiently sensitive chemical methods required to identify the substances used by insects for chemical communication, many trail pheromones have been identified and studied (Attygalle \& Morgan, 1985; Jackson \& Morgan, 1993; Kohl et al., 2003). According to Wilson (1971, p. 249), the odour trail is the most elaborate of all known forms of chemical communication, and the complex social organization of leaf-cutting ants makes the study of their trail pheromones particularly interesting.

The first trail pheromone substance identified in leafcutting ants was methyl 4-methylpyrrole-2-carboxylate (M4MPC) (A) (Fig. 1) (Tumlinson et al., 1971). Later the same substance was identified as the pheromone in Atta cephalotes (Riley et al., 1974), Acromyrmex octospinosus (Cross et al., 1982), and Acromyrmex subterraneus subterraneus (do Nascimento et al., 1994). On the other hand, 3-ethyl-2,5-dimethylpyrazine (EDMP) (B) (Fig. 1) was identified as trail pheromone of Atta sexdens rubropilosa (Cross et al., 1979) and Atta sexdens sexdens (Evershed \& Morgan, 1983). Using older gas chromatographic techniques with packed columns, Evershed \& Morgan (1983) showed only M4MPC was found in $A$. octospinosus, but that both EDMP and M4MPC were present in workers of $A$. cephalotes, $A$. sexdens sexdens and $A$. sexdens rubropilosa, although the ratio differed considerably, with the compound that had been identified as the trail pheromone in that species present in much larger proportion. A ratio for EDMP : M4MPC of 14 : 1 was found for $A$. sexdens sexdens. Later Billen et al. (1992) showed that workers of $A$. sexdens sexdens followed artificial trails made from EDMP and M4MPC in the ratio of 14 : 1 better than trails of either of these compounds alone. In trail-following tests with A. subterraneus subterraneus do Nascimento et al. (1994) tested combinations of dimethylpyrazine (C), trimethyl pyrazine (D) (Fig. 1), EDMP and M4MPC, but found no activity except in M4MPC, the only volatile substance found in the venom reservoirs of this species.

Atta ants show much greater diversity of size than do Acromyrmex ants. Wilson (1980) classified Atta workers into minors, mediums, major and soldier castes, dependent upon head width. Schoeters \& Billen (1990) found differences in the structure of the secretory part of the venom glands in different castes. We would like to know more about the way the composition of the volatile compounds of the venom which contains the trail pheromone varies with worker size, and to learn how the different castes behave towards the trail pheromone. We report here on caste differences in the deposition and following of trail pheromone by $A$. sexdens sexdens workers.

\section{MATERIAL AND METHODS}

Atta sexdens sexdens workers used in the trail following experiments were obtained from two laboratory colonies, which originated some years ago from Cayenne, French Guyana. Both colonies were kept in interconnected plastic nest boxes, in which they cultivated their fungus. These were maintained at a temperature of $23.5^{\circ} \mathrm{C}$ and relative humidity around $80 \%$. The boxes were connected to a foraging arena of $100 \times 50 \mathrm{~cm}$, in which a daily supply of bramble leaves was offered. Occasionally, also grapefruit and oranges were offered. Workers were divided into castes by head width according to the system of 
<smiles>CCc1nc(C)cnc1C</smiles>

3-ethyl-2,5-dimethylpyrazine EDMP<smiles>Cc1cnc(C)cn1</smiles>

2,5-dimethylpyrazine<smiles>COC(=O)c1cc(C)c[nH]1</smiles>

methyl 4-methylpyrrole-2-carboxylate M4MPC<smiles>Cc1cnc(C)c(C)n1</smiles>

trimethylpyrazine<smiles>COC(=O)c1ccccc1</smiles>

methyl phenylacetate<smiles>CCOC(=O)c1ccccc1</smiles>

ethyl phenylacetate

Fig. 1. The structures of EDMP and M4MPC and other compounds monitored.

Wilson (1980): 1.2-1.95 mm, minors; $2.0-2.95 \mathrm{~mm}$, mediums; 3.0-3.95 mm, majors; $4.0-4.8 \mathrm{~mm}$, soldiers.

\section{Trail-following tests}

Trail-following tests were carried out in two ways. First, by the circular trails method of Pasteels \& Verhaeghe (1974) with circular trails of $5 \mathrm{~cm}$ radius marked on paper with the circumference marked off in $1 \mathrm{~cm}$ arcs and secondly by a Y-test. The apparatus in which the experiments were conducted is illustrated in Billen et al. (1992), and the sliding door by which individual ants were admitted to the trail test area is shown in the diagram in that paper. For both the circular and Y-tests, a solution of venom in hexane at varying concentrations was applied onto a sheet of drawing paper with a Standardgraph 0.05 drawing pen. For artificial trails, a solution in hexane of varying concentration was used. For the circular test, the number of $1 \mathrm{~cm}$ arcs of the circle walked by 30 ants was counted. The same trail was used for all 30 ants. There was no evidence of trails being reenforced with secretion, since no food was provided. Differences in the mean trail-following response between castes or treatments were analysed statistically using $t$-tests with separate variance estimates. In Y-tests, a choice between two trails was offered at a branching point, as described by Billen et al. (1992), with the position of chemicals applied to the branches being reversed for every other treatment, so there was no bias in direction. The first and common part of the trail contained half the concentration of both the trails to be tested, to avoid an abrupt change in concentration at the point of bifurcation, as illustrated in Fig. 3 in Billen et al. (1992). This proceudure was replicated with 35 to 400 ants per treatment, depending on the experiment. Trail preferences and differences in trail preferences between workers of different size were tested using one- and two-sample binomial tests.

Because of the sensitivity with which ants can detect the pheromone, the pen and any other equipment was repeatedly washed with hexane before use, and the final washings were used in a trail test. If any following activity was noted, the apparatus was again washed with hexane and tested until no activity was observed.

\section{Chemical analysis}

For chemical analysis the venom apparatus was removed from individual ants of measured head width, and the venom gland and reservoir carefully removed and placed in a soft glass capillary and sealed, as described by Morgan (1990). A total of 53 workers with head width from 1.2 to $4.8 \mathrm{~mm}$ were prepared in this way.
Linked gas chromatography and mass spectrometry (GC-MS) was carried out using a Hewlett Packard 5890 Gas Chromatograph and 5970B Mass Selective detector with HP59970C ChemStation. The column was a fused silica capillary column $(12 \mathrm{~m} \times 0.2 \mathrm{~mm})$ coated with HP-1 (methyl silicone equivalent to OV-1) with $0.33 \mu \mathrm{m}$ film thickness. The column was linked to the mass spectrometer by a deactivated fused silica capillary $(10 \mathrm{~m} \times 0.2 \mathrm{~mm})$. The carrier gas was helium at $1 \mathrm{ml} \mathrm{min}^{-1}$. The GC oven was held at $30^{\circ} \mathrm{C}$ for $2 \mathrm{~min}$, then heated at $8^{\circ} \mathrm{C} \mathrm{min}{ }^{-1}$ to $200^{\circ} \mathrm{C}$ and held there $2 \mathrm{~min}$. The mass selective detector was set either to normal scan mode or to selective ion monitoring mode. In normal mode, the ions from $\mathrm{m} / \mathrm{z} 36$ to 150 were scanned at about 1.7 scans $\mathrm{sec}^{-1}$ using $70 \mathrm{eV}$ ionization. The ions scanned in selective ion mode are given in Table 1. Samples in their glass capillaries were introduced into the GC with the solid sampling device described by Morgan (1990).

2,5-Dimethylpyrazine, trimethylpyrazine and ethyl and methyl phenylacetate (all shown in Fig. 1) were purchased from Aldrich; methyl 4-methylpyrrole-2-carboxylate from Sigma, 3-ethyl-2,5-dimethylpyrazine was synthesized in the laboratory by an unpublished method, and determined to be $99 \%$ pure by chromatography. Hexane was HPLC grade.

\section{Concentration of pheromone compounds on trails}

A solution of EDMP and M4MPC (14:1) was prepared in hexane to give a total concentration of $470 \mathrm{ng}$ in $50 \mu \mathrm{l}$, the volume of solution applied to a trail of $31.4 \mathrm{~cm}$ circumference each time, equivalent to $15 \mathrm{ng} \mathrm{cm}^{-1}$ on the trail. This solution was diluted so that concentrations of $15 \mathrm{ng} \mathrm{cm}^{-1}, 1.5 \mathrm{ng} \mathrm{cm}^{-1}$, $150 \mathrm{pg} \mathrm{cm}^{-1}, 15 \mathrm{pg} \mathrm{cm}^{-1}$ and $1.5 \mathrm{pg} \mathrm{cm}^{-1}$ of the $14: 1$ mixture were applied to circular trails and the mean number of arcs walked by 30 median ants (head width $2.0 \pm 0.2 \mathrm{~mm}$ ) was noted. First, two blank tests were performed. The first with just the circle drawn with a pencil on paper, the second with pure hexane applied to the trail. The hexane control is included with the results as zero concentration of pheromone.

TABLE 1. The characteristic ions of the substances scanned in selective ion monitoring of venom reservoirs of workers of $A$. sexdens sexdens.

\begin{tabular}{lccc}
\hline Compound & $\begin{array}{c}\mathrm{T}_{\mathrm{R}} \text { of stan- } \\
\text { dard (min) }\end{array}$ & $\begin{array}{c}\text { Start time } \\
\text { for scanning }\end{array}$ & $\begin{array}{c}\text { Characteristic } \\
\text { ions scanned }\end{array}$ \\
\hline Dimethylpyrazine & 8.0 & 7.0 & $42,79,108$ \\
Trimethylpyrazine & 9.0 & 8.0 & 44,122 \\
EDMP & 10.9 & 9.8 & $42,56,135,136$ \\
Methyl phenylacetate & 12.5 & 11.5 & 91 \\
M4MPC & 13.6 & 12.5 & $53,79,108,139$ \\
\hline
\end{tabular}




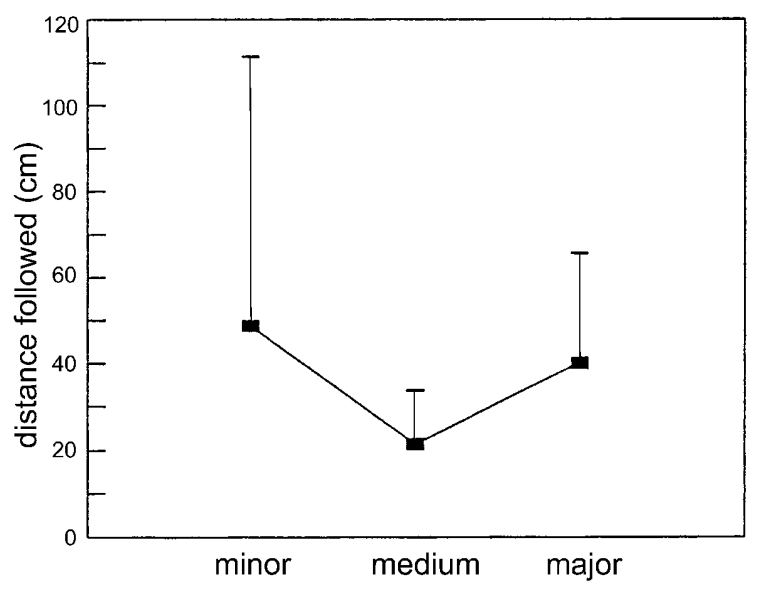

Fig. 2. Comparison of the ability of minors, mediums and majors to follow a trail made from a hexane extract of two venom glands of medium workers dissolved in hexane and applied to a circular trail (means with standard deviation, $n=30$ ants per trial).

To test the preference of ants for trails with different concentrations, Y-tests were used. Concentrations of $15 \mathrm{pg} \mathrm{cm}^{-1}$ per trail of the mixed compounds on one branch against $150 \mathrm{pg} \mathrm{cm}^{-1}$ on the other, $150 \mathrm{pg} \mathrm{cm}^{-1}$ against $1.5 \mathrm{ng} \mathrm{cm}^{-1}$ and $1.5 \mathrm{ng} \mathrm{cm}^{-1}$ against $15 \mathrm{ng} \mathrm{cm}^{-1}$ were each tested with 400 ants.

\section{Width of synthetic trails}

In order to determine the effect of width of trails on workers, linear trails, $15 \mathrm{~cm}$ long, of different breadth $(3,5,6,7,8,10$ and $12 \mathrm{~mm}$ ) were laid on paper by repeatedly drawing the pen over the paper, guiding it by a ruler and moving the ruler sideways $1 \mathrm{~mm}$ each time to give the necessary breadth. The solution of pheromone giving $15 \mathrm{pg} \mathrm{cm}^{-1}$ was used. The behaviour of 35 minor ants following each of these trails were observed and recorded.

\section{Variation of pheromone content with worker size}

The amount of the compounds 2,5-dimethylpyrazine, trimethylpyrazine, 3-ethyl-2,5-dimethylpyrazine (EDMP), methyl phenylacetate, ethyl phenylacetate and methyl 4-methyl-2pyrrole carboxylate (M4MPC) (Fig. 1), all reported to be present in the venom glands of Atta sexdens, were measured in the glands of workers and soldiers of head width from $1.2 \mathrm{~mm}$ to $4.8 \mathrm{~mm}$. Because the amounts of pheromone compound are very low compared with the sensitivity of the gas chromatograph with the mass spectrometer as detector, the more sensitive method of selective ion monitoring was used. Groups of ions (listed in Table 1) were used for each compound in turn. Standard curves of amount against chromatographic peak area were prepared for each of the compounds, using the synthetic standards.

\section{RESULTS}

\section{Following ability of different castes}

Circular trails made from the contents of two venom glands of medium workers (head width $2.0 \pm 0.2 \mathrm{~mm}$ ) in $75 \mu 1$ of hexane were used. Glands of medium workers were used because they are the category that is in charge of the outside foraging and therefore should be the natural trail layers (Wilson, 1980). The results in Fig. 2 show that medium workers followed the trail least well on average, but with greatest reproducibility (mean of $21 \pm 13 \mathrm{~cm}, n=$
TABLE 2. Results of Y-test, comparing the preference, expressed as \%, of three categories of workers for trails of different concentration (in pg or $\mathrm{ng} \mathrm{cm}^{-1}$ ). For each trial, 400 ants were tested; nd - not determined.

\begin{tabular}{|c|c|c|c|}
\hline \multirow{2}{*}{ Caste } & \multicolumn{3}{|c|}{ Concentration of trail in Y-branch } \\
\hline & $15 \mathrm{pg}: 150 \mathrm{pg}$ & $150 \mathrm{pg}: 1.5 \mathrm{ng}$ & $1.5 \mathrm{ng}: 15 \mathrm{ng}$ \\
\hline Minor & nd nd & 15.584 .5 & 20.579 .5 \\
\hline Medium & 9.290 .8 & 17.582 .5 & 21.278 .8 \\
\hline Major & nd nd & 26.573 .5 & 20.579 .5 \\
\hline
\end{tabular}

30). The minor workers followed the trail for a greater distance before losing it, but their ability was very variable between individuals ( $49 \pm 62 \mathrm{~cm}, n=30)$. The major workers were intermediate in both respects $(40 \pm 25 \mathrm{~cm}, n$ $=30$ ). On average, minor workers followed the trail for a significantly greater distance than medium workers $(t=$ 2.38 , d.f. $=31, p=0.02$ ), and major workers followed the trail for a significantly greater distance than mediums $(t=$ 3.64 , d.f. $=41, p=0.0007)$. The trail following ability of minors and majors was not significantly different $(t=$ 0.72 , d.f. $=38 p=0.48$ ).

\section{Concentration of pheromone compounds on trails}

The ability of median workers to follow circular trails of different concentration was examined. The results are shown in Fig. 3. Optimum trail following was produced by concentrations between $15 \mathrm{pg} \mathrm{cm}^{-1}$ and $150 \mathrm{pg} \mathrm{cm}^{-1}$. Trails with a lower or higher concentration than this optimum range appeared to elicit poorer following activity. All differences in trail following on substrates with varying concentrations of trail pheromone were highly significant ( $t$-test, $n=30$ ants per trial, $p<0.0002$ ), except between the categories with 15 and $150 \mathrm{pg} \mathrm{cm}^{-1}$ and with 1.5 and $15 \mathrm{ng} \mathrm{cm}^{-1}$ of trail pheromone ( $t$-test, $p>0.05)$.

To test the workers' preference for different concentrations of trail pheromone in another way, ants from the three categories minor, medium and major workers were

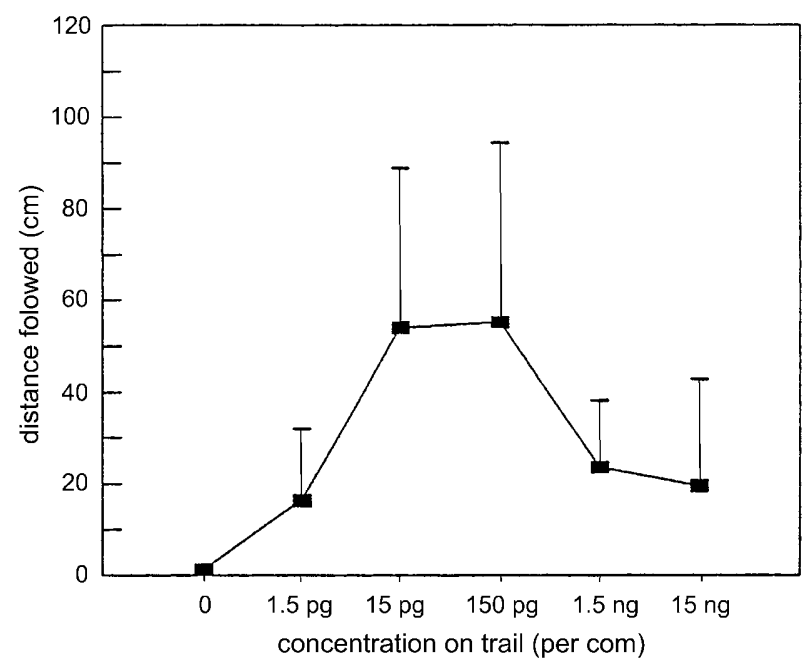

Fig. 3. The ability of medium workers to follow circular trails of different concentrations (means with standard deviations, $n=$ 30 ants per trial). The zero concentration is a hexane control. 

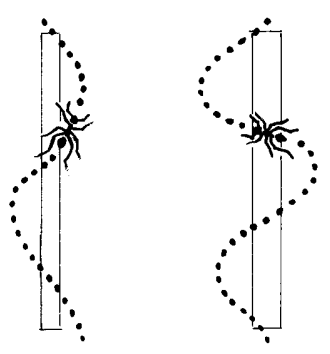

3

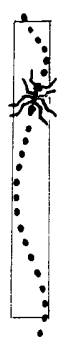

6

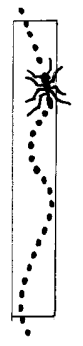

7

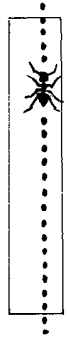

8

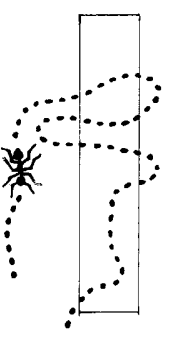

10

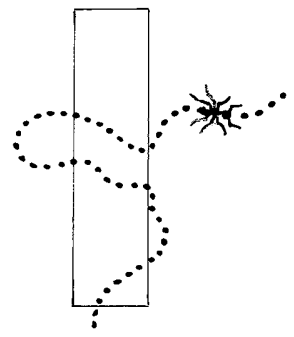

12

Fig. 4. The behaviour of minor ants on artificial trails of differing width. The number beneath each diagram is the width of the trail in $\mathrm{mm}$.

subjected to a Y-test bioassay. The results are given in Table 2. In all cases, at the branching point, the more concentrated trail was preferred to the extent of at least $70 \%$, and in all cases the results showed a highly significant bias away from a $50: 50$ ratio (one-sample binomial tests, $p$ in all cases $\left.<1 \times 10^{-19}\right)$. The preference of different castes of worker for trails of differing concentration was also tested with the same Y-test. Minor, medium and major workers did not show any significant differences in their preference for the more concentrated trail in the 1.5 vs. $15 \mathrm{ng} \mathrm{cm}^{-1}$ trials (two-sample binomial tests, $p$ in all cases > 0.79). In the $150 \mathrm{pg}$ vs. $1.5 \mathrm{ng} \mathrm{cm}^{-1}$ trials, however, the minor and medium workers showed a significantly stronger preference for the more concentrated trail than major workers (two-sample binomial test, medium vs. major: $p=0.002$, minor vs. major: $p=0.0001$ ).

\section{Width of synthetic trails}

The ants followed trails up to $9 \mathrm{~mm}$ wide, but more than $9 \mathrm{~mm}$ they could not reliably follow. The results were not quantified, but their behaviour is illustrated in Fig. 4.

\section{Nest specificity of trails}

An extract of two venom glands of medium workers in hexane was tested on medium workers from the same colony and on similar workers from a different colony in a Y-test. For each test, the choice of 35 workers at the branching point was recorded. When testing the venom from Colony 1, 26 ants followed the branch with their own colony's venom, and 9 chose the branch with venom from the foreign colony. There was therefore a significant preference for their own trails among ants from this colony (one-sample binomial test, $p=0.004$ ). When the ants from colony 2 were given a choice, 17 chose the trail with the venom of colony 1 , and 18 chose the trail with

TABLE 3. Effect of addition of extract of Dufour glands on the number of ants choosing that branch of the trail in a Y-test in two trials.

\begin{tabular}{lcc}
\hline & \multicolumn{2}{c}{ Extract of } \\
\cline { 2 - 3 } & Venom gland & Venom + Dufour gland \\
\hline 1st trial & 18 & 17 \\
2nd trial & 17 & 18 \\
\hline
\end{tabular}

their own venom, so no preference was found (onesample binomial test, $p=0.87$ ).

\section{Influence of the Dufour gland}

To see if the Dufour gland had any effect on trail following, two replicates of the Y-test were made where a hexane extract of the venom sac of two medium workers was placed on one arm of the Y-shaped trail and a hexane extract of two Dufour glands and two venom sacs was placed on the other arm. For each test the response of 35 medium workers as they reached the branching point was observed. The results are given in Table 3 . In both trials, the Dufour gland was found to have no effect on the choice of trail (one-sample binomial test, $p=0.87$ for both trials).

\section{Variation of pheromone content with worker size}

The amount of 2,5-dimethylpyrazine (DMP), trimethylpyrazine (TMP), 3-ethyl-2,5-dimethylpyrazine (EDMP), methyl phenylacetate (MPA), ethyl phenylacetate (EPA, Fig. 1) as well as methyl 4-methylpyrrole-2carboxylate (M4MPC) present in venom glands of workers of varying size is shown in Table 4. Each of the pyrazines (DMP, TMP and EDMP) were present in a significantly higher concentration in workers of increasing size as shown by pairwise $t$-tests (e.g. minors vs. majors:

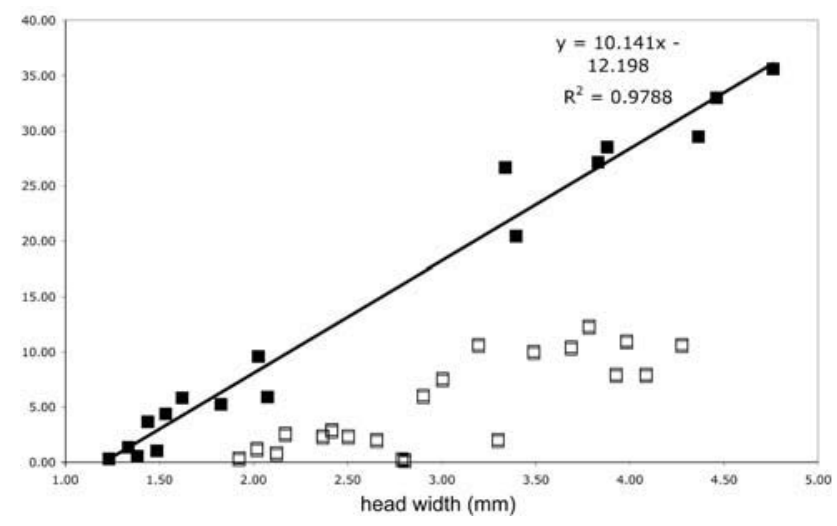

Fig. 5. The correlation between worker size as measured by head width and the amount of EDMP present in the venom gland. Filled squares represent glands dissected intact, open squares represent glands from which some secretion may have been lost during dissection. The regression line applies to the filled squares. 
TABLE 4. The amount (ng per gland $\pm \mathrm{SD}$ ) of volatile compounds in the venom of workers of $A$. sexdens sexdens of different size groups.

\begin{tabular}{lcccc}
\hline & \multicolumn{4}{c}{ Worker caste (head width and number of samples) } \\
\cline { 2 - 5 } Compound & Minors & Mediums & Majors & Soldiers \\
& $(1.2-1.9 \mathrm{~mm}, \mathrm{n}=11)$ & $(2.0-2.9 \mathrm{~mm}, \mathrm{n}=14)$ & $(3.0-3.9 \mathrm{~mm}, \mathrm{n}=13)$ & $(4.0-4.8 \mathrm{~mm}, \mathrm{n}=6)$ \\
\hline DMP & $0.29 \pm 0.18$ & $0.41 \pm 0.20$ & $0.60 \pm 0.21$ & $0.58 \pm 0.21$ \\
TMP & $0.38 \pm 0.17$ & $0.48 \pm 0.15$ & $0.62 \pm 0.23$ & $1.38 \pm 0.42$ \\
EDMP & $2.56 \pm 1.94$ & $3.28 \pm 2.51$ & $13.25 \pm 9.20$ & $18.27 \pm 12.58$ \\
MPA & 0 & 0.2 & $0.84 \pm 0.53$ & 1.1 \\
EPA & 0 & 0.1 & $0.25 \pm 0.21$ & 0 \\
M4MPC & $0.06 \pm 0.05$ & $0.14 \pm 0.09$ & $0.20 \pm 0.18$ & $0.04 \pm 0.02$ \\
\hline
\end{tabular}

$p<0.007$ for all 3 compounds). The amount of EDMP that was present showed the strongest correlation with the size of the workers, as measured by their head width (Pearson $\mathrm{R}^{2}=0.98, n=17, p<1 \times 10^{-13}$ ). MPA showed a similar trend. EPA and M4MPC were present in small amounts in majors, but were not detectable in minors and found in measurable amounts in only two mediums and one soldier. The amount of EDMP found is plotted against head width in Fig. 4. During dissection of the glands, in some cases some secretion was possibly lost, these are shown as open squares, while the filled squares show those where the gland was removed undamaged.

\section{DISCUSSION}

The optimum amount of pheromone on a trail for $A$. sexdens sexdens $\left(15-150 \mathrm{pg} \mathrm{cm}^{-1}\right)$ can be compared with that observed for Myrmica rubra (3-300 $\mathrm{pg} \mathrm{cm}^{-1}$ of EDMP, Evershed et al., 1982), which was determined by the same method. When given a choice to decide between two trails with different concentrations in a Y-test, workers preferred the more concentrated trail. When tested on trails with various concentrations separately (the circle test), however, the ants do not react well to high concentrations. At the branching point, the ants are able to detect the higher concentrations, but in prolonged following, we conclude their senses become overloaded and their ability to follow the scent falls. We have shown with both Myrmica rubra (Evershed et al., 1982) and Tetramorium caespium (Attygalle \& Morgan, 1983) there is an optimum concentration of trail following. The sensitivity of medium $A$. sexdens sexdens workers would appear to be similar to, or more sensitive than, that of M. rubra workers in following trails.

Hangartner (1967) and Wilson (1971, p. 250) have explained the behaviour of ants following a trail as moving in a tunnel of pheromone vapour. Hangartner (1967) showed that the ant turns from side to side testing the walls of the vapour tunnel with its antennae. If the vapour tunnel is too narrow, less than the reach of the antennae, they are constantly moving outside the tunnel and the ant has difficulty keeping inside it. If the tunnel is too wide, the ant is unable to register both sides and finds itself lost in a broad cavern rather than in a tunnel. Wilson and Bossert (1963) in an early theoretical consideration of the trail pheromone of the fire ant Solenopsis invicta suggested the width of the odour tunnel would need to be 1 $\mathrm{cm}$ wide for optimum following by workers of this spe- cies. We found that a single pass of the pheromone solution on a trail is sufficient for $A$. s. sexdens minor workers to follow. By using the minimum concentration for optimum trail-following, and making repeated passes with the solution, displaced sideways each time by $1 \mathrm{~mm}$, we avoided concentration effects, since concentrations up to $150 \mathrm{pg} \mathrm{cm}^{-1}$ are not statistically different (Fig. 3). It is clear by the behaviour of the ants that they could detect the width of the trail (Fig. 4). Beyond $8 \mathrm{~mm}$ the ants lost the direction of the trail, as suggested by Hangartner (1967). It is difficult to relate the width of the trails we deposited to the actual space occupied by pheromone vapour above them, but one would expect diffusion to make the vapour space wider than the trail laid, and Wilson and Bossert's value may not be far out.

The increase in amount of EDMP with body size illustrated in Fig. 4 agrees with that found by Evershed \& Morgan (1983), although higher values for the largest ants were found here. Difficulties over the determination of M4MPC by gas chromatography leave the ratio of EDMP to M4MPC unclear. The value of 14:1 given by Evershed \& Morgan (1983) was obtained from peak areas only, with less well resolved chromatograms, so the peaks may have included other compounds. Here the EDMP peaks were well resolved and easily quantified, but the M4MPC peaks showed tailing on the non-polar column, so that integration of very small trailing peaks was not accurate. An overall ratio of EDMP : M4MPC of $100: 1$ is obtained on these results. It is highly likely that the amounts of M4MPC recorded here are underestimated.

Cross et al. (1979) found methyl and ethyl phenylacetate accompanied the EDMP in Atta sexdens rubropilosa. Therefore we monitored all the samples analysed here for these compounds as well. We found methyl phenylacetate in less than one quarter of the samples and the ethyl ester in only three (Table 4). We conclude this is another difference between the two subspecies. The presence of dimethylpyrazine and trimethylpyrazine in small but relatively constant amount has been a new discovery. In some species (e.g. Tetramorium caespitum) both 2,5-dimethylpyrazine and EDMP together comprise the trail pheromone (Attygalle \& Morgan, 1983), while in others (e.g. Manica rubida, where both the dimethyl- and trimethylpyrazines are present) EDMP alone is the active compound (Attygalle et al., 1986). No information is yet available on the activity, or lack of it, of dimethyl- and trimethyl-pyrazines on Atta sexdens sexdens trails. 
ACKNOWLEDGEMENTS. We thank M. Bazire-Benazet for the A. s. sexdens colonies. Funding for this work was obtained through the research network INSECTS of the Universities of Copenhagen, Firenze, Helsinki, Keele, Lausanne, Leuven, Oulu, Regensburg, Sheffield, Würzburg and ETH Zürich, financed by the European Commission via the Training and Mobility of Researchers (TMR) programme. We thank E. Schoeters for help in designing the figures, and a diligent referee for careful reading of our manuscript.

\section{REFERENCES}

Attygalle A.B. \& Morgan E.D. 1983: Trail pheromone of the ant Tetramorium caespitum (L.). Naturwissenschaften 70: 364-365.

Attygalle A.B. \& Morgan E.D. 1985: Ant trail pheromones. Adv. Insect Physiol. 18: 1-30.

Attygalle A.B., Cammaerts M.-C., Cammaerts R. \& Morgan E.D. 1986: Chemical and ethological studies of the trail pheromone of the ant Manica rubida (Hymenoptera: Formicidae). Physiol. Entomol. 11: 125-132.

Billen J., Beeckman W. \& Morgan E.D. 1992: Active trail pheromone compounds and trail following in the ant Atta sexdens sexdens (Hymenoptera: Formicidae). Ethol. Ecol. Evol. 4: 197-202.

BonNET C. 1779: Observations XLIII. Sur un procédé des fourmis. In: Oeuvres d' Histoire naturelle et de Philosophie. Vol. 1. Samuel Fauché, Neuchâtel, pp. 535-536.

Cross J.H., Byler R.C., Ravid U., Silverstein R.M., Robinson S.W., BaKer P.M., de Oliveira J.S., Jutsum A.R. \& Cherrett J.M. 1979: The major component of the trail pheromone of the leaf cutting ant Atta sexdens rubropilosa Forel: 3-ethyl-2,5-dimethylpyrazine. J. Chem. Ecol. 5: 187-204.

Cross J.H., West J.R., Silverstein R.M., Jutsum A.R. \& CherRETT J.M. 1982: Trail pheromone of the leaf-cutting ant Acromyrmex octospinosus (Reich) (Formicidae: Myrmicinae). $J$. Chem. Ecol. 8: 1119-1124.

Evershed R.P. \& Morgan E.D. 1983: The amounts of trail pheromone substance in the venom of workers of four species of attine ants. Insect Biochem. 13: 469-474.
Evershed R.P., Morgan E.D. \& Cammaerts M.-C. 1982: 3-Ethyl-2,5-dimethylpyrazine, the trail pheromone from the venom of eight species of Myrmica ants. Insect Biochem. 12: 383-391.

HANGARTNER W. 1967: Spezifität und Inaktivierung des Spurpheromons von Lasius fuliginosus Latr. und Orientierung der Arbeiterinnen im Duftfeld. Z. Vergl. Physiol. 57: 103-136.

JACKSON B.D. \& MORGAN E.D. 1993: Insect chemical communication. Chemoecology 4: 125-144.

Kohl E., Hölldobler B. \& Bestmann H.-J. 2003: Trail pheromones and Dufour gland contents in three Camponotus species (C. castaneus, C. balzani, C. sericeiventris: Formicidae, Hymenoptera). Chemoecology 13: 113-122.

Morgan E.D. 1990: Preparation of small-scale samples from insects for chromatography. Anal. Chim. Acta 236: 227-235.

Nascimento R.R. Do, Morgan E.D., Moreira D.O. \& Della LuCIA T.M.C. 1994: Trail pheromone of leaf-cutting ant Acromyrmex subterraneus subterraneus (Forel). J. Chem. Ecol. 20: $1719-1724$

Pasteels J.M. \& Verhaeghe J.C. 1974: Dosage biologique de la phéromone de piste chez les fourrageuses et les reines de Myrmica rubra. Insectes Soc. 21: 167-179.

Riley R.G., Silverstein R.M., Carroll B. \& Carroll R. 1974: Methyl 4-methylpyrrole-2-carboxylate, a volatile trail pheromone from the leaf-cutting ant Atta cephalotes. J. Insect Physiol. 20: 651-654.

Schoeters E. \& Billen J. 1990: Morphology of the venom gland in relation to worker size in leaf-cutting ants. Actes Colloq. Insectes Soc. 6: 249-252.

Tumlinson J.H., Silverstein R.M., Moser J.C., Brownlee R.G. \& RUTH J.M. 1971: Identification of the trail pheromone of a leaf-cutting ant Atta texana. Nature 234: 348-349.

WILson E.O. 1971: The Insect Societies. The Belknap Press of Harvard University Press, Cambridge, MA, 548 pp.

WILSON E.O. 1980: Caste and division of labor in leaf-cutting ants (Hymenoptera: Formicidae: Atta) I. The overall pattern in Atta sexdens. Behav. Ecol. Sociobiol. 7: 143-156.

WiLSON E.O. \& Bossert W.H. 1963: Chemical communication among animals. Rec. Progr. Hormone Res. 19: 673-716.

Received October 13, 2005; revised and accepted January 24, 2006 